\title{
Algoritmo basado en Árboles de decisiones para la predicción óptima del Sistema Pensionario Peruano: Revisión sistemática de la literatura
}

\section{Algorithm based on Decision Trees for the optimal prediction of the Peruvian Pension System: Systematic review of the literature state, Venezuela}

\author{
José Antonio Ogosi Auqui ${ }^{1}$, Luis Miguel Romero Echevarría ${ }^{2}$ y Jorge Víctor Mayhuasca Guerra ${ }^{1}$ \\ 'Universidad Nacional Federico Villarreal, Perú. \\ ${ }^{2}$ Universidad Nacional Autónoma de Tayacaja Daniel Hernández Morillo, Perú.
}

\begin{abstract}
RESUMEN
En el presente artículo se ha desarrollado teniendo en cuenta las directrices para la Revisión Sistemática de la Literatura (SLR), se centra en lo fundamental que es la elección de un seguro de pensiones, teniendo en cuenta que existen dos tipos sistemas: El Sistema Privado de Pensiones (AFP), en el que el trabajador recibe lo que ha cobrado a lo largo del período que ha trabajado como una cuenta de ahorro personal; y el Seguro Nacional de Pensiones (ONP), que es un organismo público que asigna el dinero aportado por los trabajadores (fondo común) y se desembolsa como concepto de pensiones a los jubilados. Teniendo en cuenta el problema de la falta de información sobre el Seguro de Pensiones que brindan tanto las entidades públicas como privadas, se decidió construir un algoritmo de árbol decisión para de acuerdo a una cierta información brindada se pueda lograr una toma de decisiones relevante para cada trabajador que acceda a planilla por primera vez o para aquellas personas que trabajan de forma independiente, ya que esta decisión será fundamental para la futura estabilidad económica del trabajador.
\end{abstract}

Palabras clave: árbol de decisión, sistema privado de pensiones, sistema nacional de pensiones, trabajador activo, trabajador independiente, algoritmo.

\section{Abstract}

This article details how fundamental pension insurance is, taking into account that there are two systems: The Private Pension System (AFP), in which the worker receives what he has collected throughout the period he has worked. , such as a personal savings account and the National Pension Insurance (ONP), which is a public body that allocates the money contributed by workers (common fund) and is disbursed as a concept of pensions to retirees. Taking into account the problem of the lack of information on Pension Insurance that both public and private entities provide, it was decided to build an algorithm for adequate information and thus achieve a relevant decision-making for each worker who accesses to payroll for the first time or for those people who decide to start independently, since this decision will be fundamental for the future economic stability of the unemployed worker.

Keywords: decision tree, private pension system, national pension system, active worker, independent worker, algorithm.

José Antonio Ogosi Auqui. ORCID: https://orcid.org/0000-0002-4708-640X email: jogosi@unfv.edu.pe Luis Miguel Romero Echevarría. ORCID: https://orcid.org/0000-0002-1693-2115 email: luisromero@unat.edu.pe Jorge Víctor Mayhuasca Guerra. ORCID: https://orcid.org/0000-0002-6465-4738 email: jmayhuasca@unfv.edu.pe 


\section{INTRODUCCIÓN}

A menudo cuando un trabajador comienza su vida laboral dentro de una empresa, carece de conocimiento o no se encuentra correctamente informado sobre cuál es la opción ideal o qué opción le genera un mayor beneficio cuando tenga que decidir sobre su seguro de pensiones, por consiguiente es designado de acuerdo el criterio del empleador, generando de esta forma inconvenientes futuros; puesto que al querer realizar una modificación de seguro de pensiones podría ser adverso para el trabajador, principalmente cuando se desea llevar a cabo una modificación de un seguro nacional a un seguro privado.

Poco más del $25 \%$ de los peruanos dispone de un seguro. Una gran cantidad de trabajadores asalariados e independientes no efectúa aportes con regularidad. Y un tercio de aquellos que sí dispone de un seguro posiblemente obtendrán una pensión inferior al mínimo vital cuando llegue el momento de su jubilación. A diario se conoce mayores casos de personas de la tercera edad que no pueden vivir decentemente, puesto que la pensión que obtiene no les satisface ni para cubrir sus medicamentos.

El ciudadano peruano que ha contribuido tanto en el SNP como el SPP se ha encontrado básicamente olvidado a su suerte en relación las decisiones a tomar respecto de su pensión. Por encima de los reportes de estado de cuenta (que no precisan de información clave como, por ejemplo, la estimación de la pensión que el trabajador puede aspirar) y las aproximaciones agresivas de las fuerzas de venta, no se ha producido una asesoría activa e independiente que le adviertas de decisiones inadecuadas.

El presente artículo tiene como objetivo garantizar que las personas seleccionen el régimen de Sistema Pensionario más apropiado en concordancia con la coyuntura de su condición como trabajador que accede a planilla. Por consecuencia, se está decidiendo por el desarrollo de un algoritmo con el propósito de elegir apropiadamente los Seguros Pensionarios, como una solución a la ausencia de información de las personas que comienzan su vida laboral en planilla proporcionando saberes preliminares sobre los tipos de seguros existentes, así orientarlo para la adecuada elección de su seguro pensionario y así prevenir dificultades económicas futuras. Los resultados alcanzados del presente artículo posibilitarán comprobar la legitimidad y seguridad de la aplicación del marco metodológico, al igual que de los instrumentos empleados. Una vez ratificada la legitimidad, seguridad de los métodos; los procedimientos, técnicas e instrumentos que se utilizaron en la presente investigación permitirán ser aplicados en otros estudios semejantes en la cual se busque contribuir al campo de desarrollo de Sistemas Cognitivos

\section{Metodología}

El presente método de revisión se ha desarrollado teniendo en cuenta las directrices de B. Kitchenham para la Revisión Sistemática de la Literatura (SLR), un estudio para mapear, identificar, evaluar críticamente, consolidar y recopilar los resultados de estudios primarios relevantes sobre un tema de investigación determinado. La SLR se convierte en un método estándar para obtener una respuesta mediante la realización de una revisión de la literatura basada en los estudios anteriores relevantes. El propósito de realizar SLR es resumir la investigación previa, identificar la brecha que se necesita llenar entre la investigación anterior y la actual, producir una síntesis de informe coherente y hacer un marco de investigación. El método de revisión elabora las preguntas de investigación, las fuentes y estrategia de búsqueda, los criterios de selección, la selección de estudios, la evaluación de la calidad, la extracción de datos y la síntesis de datos. El documento está organizado de la siguiente manera. La sección II es el background de la investigación. La sección III detalla la metodología de revisión. La sección IV presenta los Resultados y discusiones. Finalmente, la sección V presenta las conclusiones y futuras investigaciones.

\section{Preguntas de investigación}

El objetivo de la SLR es aclarar y evaluar la evidencia empírica resultante de varios estudios donde se aplica el Algoritmo basado en Árboles de decisión. Por lo cual, se ha formulado $y$ analizado las siguientes preguntas de investigación (RQ): RQ1. ¿Qué tipo de algoritmo de machine learning se ha empleado para la predicción del sistema pensionario? RQ2. ¿Qué impacto han tenido la aplicación de algoritmo de árbol de decisión en la predicción del sistema pensionario para los trabajadores iniciales en planilla? RQ3. ¿Qué tipos de variables se utilizan para realizar el análisis y procesamiento del algoritmo de decisión para la predicción del sistema pensionario?

En la Tabla 1 se observa los objetivos de investigación de este estudio a través de la correspondencia entre cada pregunta de investigación y cada motivación.

\section{Tabla 1.}

Objetivos de la investigación.

\section{Pregunta de investigación}

\section{Motivación}

$\begin{array}{lll}\text { RQ1. ¿Qué tipo de } & \text { Identificar cuáles son los } \\ \text { algoritmo de machine } & \text { tipos de algoritmos de } \\ \text { learning se ha empleado } & \text { machine learning son } \\ \text { para la predicción del } & \text { empleados } & \text { para } \\ \text { sistema pensionario? } & \text { predicción. }\end{array}$

RQ2. ¿Qué impacto han tenido la aplicación de algoritmo de árbol de decisión en la predicción del sistema pensionario para los trabajadores iniciales en planilla?

RQ3. ¿Qué tipos de Identificar aquellas variables se utilizan para variables que son realizar el análisis y necesarias y procesamiento del fundamentales para algoritmo de decisión para realizar un óptimo la predicción del sistema proceso de predicción pensionario? del sistema pensionario. 
ISSNe: 2617-9156

\section{Fuentes de búsqueda y estrategias de búsqueda}

Para obtener información de calidad para la investigación, se investigó sobre la existencia de repositorios, revistas, buscadores; orientados a el almacenamiento y búsqueda de publicaciones de carácter científico.

De todos los repositorios y buscadores encontrados, se seleccionó los más destacados; dentro de dicha la lista se tiene: IEEE Xplore, Dialnet, Science Direct, ResearchGate, Wiley Online Library. La lista mencionada se conformó por buscadores y repositorios que se consideran de mayor prestigio en el ámbito de publicaciones de carácter científico. Si bien se menciona que los repositorios elegidos son de prestigio, es necesario conocer cómo funcionan estos repositorios. Se sabe que para una mejor búsqueda se debe tener en cuenta que cada buscador, tiene su ecuación de búsqueda para realizar búsquedas avanzadas. Para formular dichas ecuaciones se necesitan términos de búsqueda, en este caso, las variables (dependiente, independiente e interviniente). La Tabla 2 refleja los términos de búsqueda mencionado anteriormente.

\section{Tabla 2.}

Términos de búsqueda.

\begin{tabular}{lll}
\hline \multicolumn{3}{c}{ Descriptor } \\
\hline \multicolumn{1}{c}{ Español } & \multicolumn{1}{c}{ Inglés } \\
\hline $\begin{array}{l}\text { Algoritmo basado en árboles de } \\
\text { decisión }\end{array}$ & $\begin{array}{l}\text { Decision tree based } \\
\text { algorithm }\end{array}$ \\
$\begin{array}{l}\text { Predicción del sistema } \\
\text { pensionario peruano }\end{array}$ & $\begin{array}{l}\text { Prediction of the } \\
\text { Peruvian } \\
\text { system pension } \\
\text { Método / Metodología /Modelo } \\
\text { Method / Methodology } \\
\text { /Model }\end{array}$ \\
\hline
\end{tabular}

Luego de conocer los términos de búsqueda, se formuló las siguientes ecuaciones de búsqueda, reflejadas en la Tabla 3.

Tabla 3.

Ecuaciones de búsqueda.

\begin{tabular}{cl}
\hline Source & \multicolumn{1}{c}{ Generic Search Equation } \\
\hline & ("All Metadata":pension) OR ("All \\
& Metadata":pension system) AND \\
("All Metadata":decision algorithm) & AND ("All Metadata":machine \\
learning) & \\
& (Pension OR pension system) AND \\
& (decision algorithm AND machine \\
Dialnet & learning) \\
& ("Pension" OR "Pensión system") \\
& AND ("Decision algorithm" AND \\
ScienceDirect & "Machine learning") \\
& (Pension OR pension system) AND \\
& (decision algorithm AND machine \\
ResearchGate & learning) \\
& "Pension OR pension system" \\
Wiley Online & anywhere and "decision algorithm \\
Library & AND machine learning" anywhere \\
\hline
\end{tabular}

Dichos descriptores de búsqueda permitieron la formulación de las ecuaciones de búsqueda genérica para cada una de las fuentes de búsqueda. Las principales ecuaciones de búsqueda genérica, así como sus fuentes y número de resultados base correspondiente, se mencionan a continuación en la Tabla 4.

\section{Tabla 4.}

Matriz consolidada del número de resultados por fuente.

\begin{tabular}{cc}
\hline Source & $\begin{array}{c}\text { Número de } \\
\text { resultados }\end{array}$ \\
\hline IEEE Xplore & 103 \\
Dialnet & 8 \\
ScienceDirect & 6 \\
ResearchGate & 100 \\
Wiley Online Library & 146 \\
\hline
\end{tabular}

Se encontró un total de 363 publicaciones que tienen relación con el tema de investigación, pero dicha cantidad de publicaciones se considera demasiado para investigar, es por eso por lo que se aplicó ciertos criterios de exclusión para seleccionar publicaciones que nos sean de mayor utilidad para la investigación.

\section{Criterios de selección}

Se planteó los siguientes criterios de inclusión y exclusión son los siguientes:

C1: Son elegibles aquellas publicaciones científicas que tengan relación con la ingeniería de sistemas y donde se traten las RSL.

C2: Los artículos no mencionan una metodología, modelo ni método.

C3: Los artículos no tienen una antigüedad menor a 5 años.

C4: Los artículos están publicados journals o conferences.

C5: Se incluyeron estudios en 2 idiomas: inglés y español.

C6: Se excluyeron paper sin diseño de investigación como workshops, encuestas u otros sin pregunta de investigación definida.

C7: Se utilizaron RSL como estudios secundarios con estructura bien definida en su abstract.

C8: No se consideraron revisiones terciarias, ni actualizaciones de revisiones.

C9: Los artículos son reconocidos como artículos científicos o tesis de postgrado.

C10: Se excluyeron las investigaciones cuyos títulos y los keywords de los artículos no son adecuados

C11: Se excluyeron los artículos carecen de suficiente información para hacer valoración.

C12: Se excluyeron los artículos cuyo abstract no es relevante.

C13: No se consideraron RSL de RSL anteriores (siguen la misma estructura). Se excluyeron protocolos de RSL.

Dichos criterios nos ayudaran a filtrar publicaciones que no aporten información útil.

\section{Evaluación de la calidad}

El paso siguiente, luego de haber realizado los criterios de selección y exclusión se determinó que se llegó a la cantidad 
ISSNe: 2617-9156

de 53 artículos científicos quienes se adecuaron a todos los criterios establecidos, posteriormente los autores llevaron a cabo criterios para evaluar la calidad de las publicaciones para asegurar que no se esté trabajando con publicaciones de poca utilidad con respecto a las preguntas de investigación establecidas anteriormente.

Se realizaron los siguientes criterios de calidad:

QA1: ¿El tema pertenece a los campos de la informática, sistemas de información?

QA2: ¿El articulo pertenece a un libro o publicación?

QA3: ¿El documento está bien organizado?

QA4: ¿Los objetivos de investigación se identifican claramente en el documento?

QA5: ¿Explica el contexto en el que se realizó la investigación?

QA6: ¿Se dispone del texto completo del documento?

QA7: En general, ¿Considera útil el documento?

La revisión de los criterios de calidad se realizó después de los criterios de exclusión, ya ayudó a filtrar los ítems seleccionados para elegir los más relevantes para la investigación. De los 53 artículos, 21 no cumplieron con los siete criterios de evaluación de la calidad. Por lo cual, se excluyeron de la investigación.

Extracción de datos

El procedimiento de extracción de datos ha sido desarrollado teniendo en cuenta cuatro propiedades importantes de los datos, planteadas en los objetivos de investigación. Estas propiedades han sido reconocidas por la literatura disponible y por los estudios seleccionados. Las cinco propiedades consideradas en la extracción son: Algoritmo basado en árboles de decisión; Metodologías/ modelos/ métodos utilizados; Algoritmo de machine Learning; Predicción del sistema pensionario.

Síntesis de datos

El proceso de síntesis de datos incluye reunir los datos y concluir las respuestas según las preguntas de investigación. La síntesis de datos se ha realizado analizando los estudios seleccionados a través de técnicas de regresión multivariante para el metaanálisis, así como las siguientes medidas estadísticas descriptivas: promedio, moda, mediana, sumatorias, conteo, porcentajes.

\section{Resultados}

RQ1. ¿Qué tipo de algoritmo de machine learning se ha empleado para la predicción del sistema pensionario?

La tabla 5 enumera cuales son los principales tipos de algoritmos de machine learning para la predicción.

Los resultados estadísticos muestran que la aplicación de algoritmos de árboles de decisión tiene el mayor porcentaje (33\%), seguida de la aplicación de otros tipos de algoritmos (27\%), posterior se tiene a los algoritmos de regresión y de redes neuronales (13\%) y por último los algoritmos bayesianos y de aprendizaje profundo (7\%).
Tabla 5.

Tipos de algoritmos de machine learning.

Tipos de algoritmos de machine learning

Cantidad

\begin{tabular}{ll}
\hline Algoritmos de regresión & 2 \\
Algoritmos bayesianos & 1 \\
Algoritmos de árbol de decisión & 5 \\
Algoritmos de redes neuronales & 2 \\
Algoritmos de aprendizaje profundo & 1 \\
Otros algoritmos & 4 \\
\hline
\end{tabular}

RQ2. ¿Qué impacto han tenido la aplicación de algoritmo de árbol de decisión en la predicción del sistema pensionario para los trabajadores iniciales en planilla?

La tabla 6 enumera cual es el impacto que tuvieron la aplicación de los algoritmos en la toma de decisiones. Los resultados estadísticos muestran que la aplicación de algoritmos de decisión tuvo un gran impacto positivo (52\%), seguida de un impacto de mayor rendimiento (20\%), posterior se tuvo un impacto de mejor eficiencia (16\%) y por último un impacto de mayor precisión (12\%).

Tabla 6.

Impacto de los algoritmos utilizados.

\begin{tabular}{lc}
\hline \multicolumn{1}{c}{ Impacto } & Cantidad \\
\hline Positivo & 13 \\
Mayor eficiencia & 4 \\
Mejor rendimiento & 5 \\
Mayor precisión & 3 \\
\hline
\end{tabular}

RQ3. ¿Qué tipos de variables se utilizan para realizar el análisis y procesamiento del algoritmo de decisión para la predicción del sistema pensionario?

La tabla 7 enumera cuales son los tipos de variables que se emplearon para el análisis y procesamiento de los algoritmos de predicción en la toma de decisiones.

Los resultados estadísticos muestran que la variable de información básica tuvo el mayor porcentaje (47\%), seguida de la variable de salud (18\%), posterior se tuvo a la variable de ingresos y otras variables (11\%), luego se consideró a la variable de trayectoria laboral (8\%) y finalmente se consideró a la variable de manutención (5\%).

\section{Tabla 7.}

Variables de procesamiento para la aplicación de los algoritmos de decisión.

\begin{tabular}{lc}
\hline \multicolumn{1}{c}{ Variables } & Cantidad \\
\hline Información básica & 18 \\
Trayectoria laboral & 3 \\
Ingresos & 4 \\
Salud & 7 \\
Manutención & 2 \\
Otras variables & 4 \\
\hline
\end{tabular}


ISSNe: 2617-9156

\section{DISCUSIóN}

En esta sección se presenta la síntesis de los hallazgos obtenidos a través de la revisión sistemática de los 25 artículos, dividido en las tres preguntas de investigación planteadas. Y después de realizar el análisis de los artículos finales, se realizó un gráfico general de estos artículos indicando la cantidad y año de subida. Observamos que el primer artículo analizado es del año 1996; asimismo, se observa que el grado de interés en aplicar estos tipos de algoritmos va en aumento a excepción del año 2018.

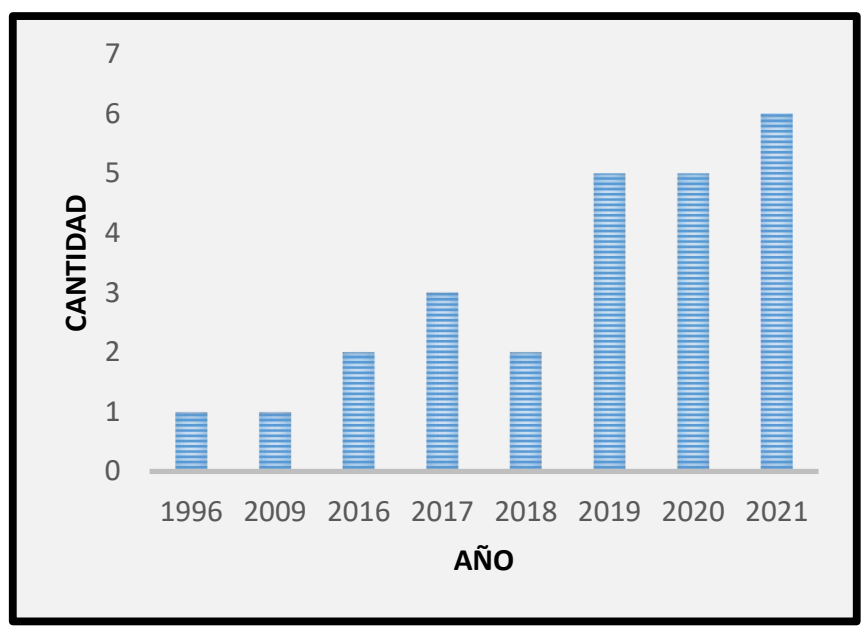

Figura 1.

Cantidad de artículos por año.

RQ1. ¿Qué tipo de algoritmo de machine learning se ha empleado para la predicción del sistema pensionario?

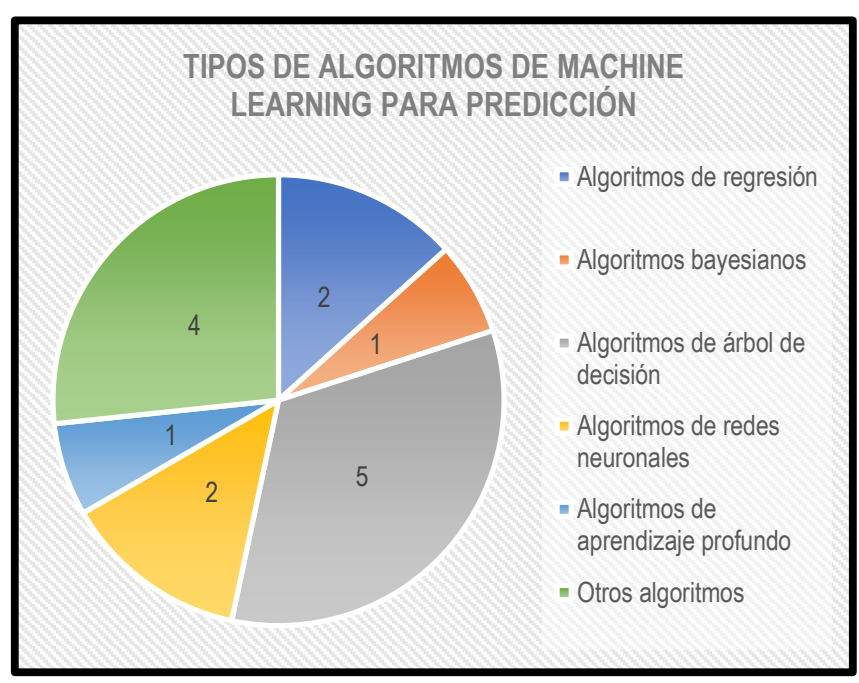

Figura 2.

Tipos de algoritmos de machine learning para la predicción.

Se puede evidenciar en la Figura 2 que el algoritmo de árbol de decisión es el tipo de algoritmo de machine learning más utilizado para la predicción en general y de un sistema pensionario. Esto se debe a que el algoritmo de árbol de decisión es un modelo predictivo que se encarga de segmentar el espacio de los predictores agrupando observaciones con valores similares para la variable respuesta o dependiente.
RQ2. ¿Qué impacto han tenido la aplicación de algoritmo de árbol de decisión en la predicción del sistema pensionario para los trabajadores iniciales en planilla?

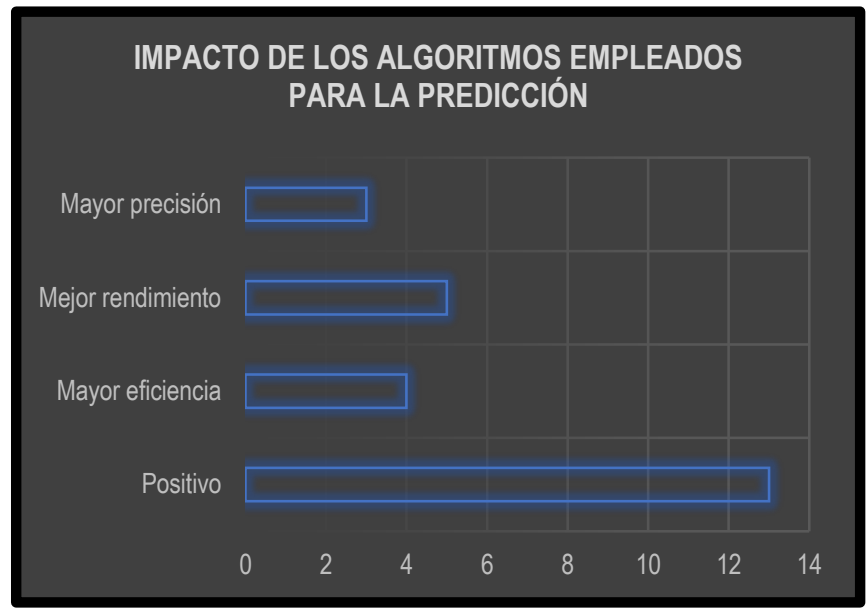

Figura 3.

Impacto de los algoritmos empleados para la predicción.

Se puede evidenciar en la Figura 3 que la aplicación de la mayoría de los algoritmos de machine learning para la predicción tienen un resultado positivo. Esto se debe a que los algoritmos de predicción permiten consolidar toda la data que se desea procesar en una única plataforma, donde el análisis y procesamiento será respaldado por la inteligencia artificial; además, permite obtener resultados más precisos y en el tiempo real.

RQ3. ¿Qué tipos de variables se utilizan para realizar el análisis y procesamiento del algoritmo de decisión para la predicción del sistema pensionario?

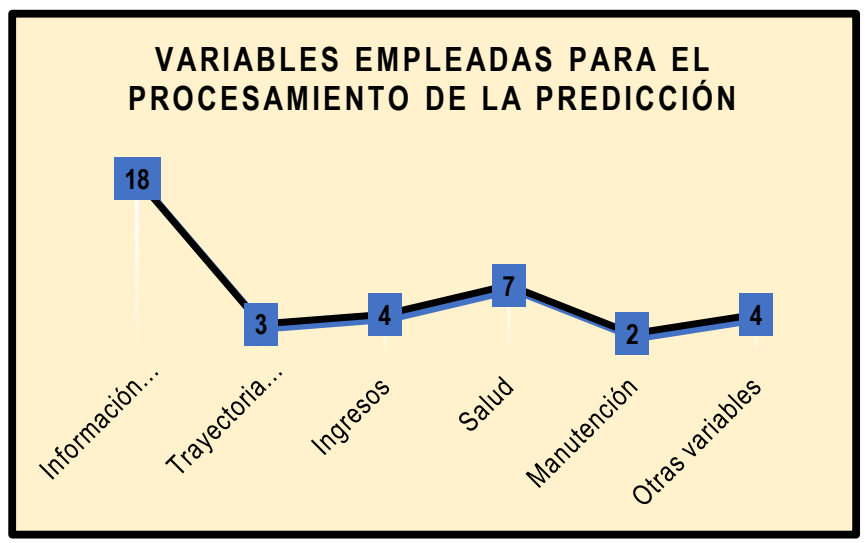

Figura 4.

Variables empleadas para el análisis y procesamiento de la predicción.

Se puede evidenciar en la Figura 4 que para el procesamiento de las variables que requiere el algoritmo de predicción es vital disponer de la información básica, ingresos y el estado de salud de los pensionistas. Esto se debe a que, conforme a la información personal brindada, el algoritmo podrá realizar el análisis y procesamiento óptimo para adecuarse a su condición en específica del trabajador. 
ISSNe: 2617-9156

\section{CONCLUSIONES}

Es fundamental que todo trabajador que accede a planilla por primera vez. o bien decida aportar de forma independiente, cuente con una herramienta que le permita seleccionar el sistema de pensión más adecuado a su condición y ello es lo que proporcionará la propuesta de Algoritmo basado en Árboles de decisiones para la elección óptima del Sistema Pensionario Peruano, el cual a través de una serie de interrogantes sobre su edad, ingresos, trayectoria laboral, salud permitirá proyectar de forma automática la alternativa más apropiada de acuerdo a la condición del trabajador.

Asimismo, está investigación permitirá ser aplicados en otros estudios semejantes a gran escala, en la cual se busque contribuir y complementar al campo de desarrollo de Sistemas Cognitivos. Donde a través de la implementación de mayores herramientas de Machine Learning potenciarán la toma de decisiones más idóneas para los trabajadores, en la cual no tan solo brinde cual sería la alternativa más conveniente, sino que proporcione mayores detalles, reportes y sugerencias sobre la situación a futuro del trabajador.

\section{REFERENCIAS}

[1] Arias-Sosa, L. A., Salamanca-Reyes, J. R., \& RamosMontaño, C. (2021). The role of different natural and human-related habitats for the conservation of birds in a high Andean Lake. Wetlands Ecology and Management, 29(6), 897-913. https://doi.org/10.1007/s11273-021-09819-3.

[2] Altman, E.I., Hotchkiss, E. and Wang, W. (2019). A 50Year Retrospective on Credit Risk Models, the Altman Z-Score Family of Models, and Their Applications to Financial Markets and Managerial Strategies. In Corporate Financial Distress, Restructuring, and Bankruptcy.

https://doi.org/10.1002/9781119541929.ch10

[3] Byrne, T, Montgomery, AE, Fargo, JD. (2019). Predictive modeling of housing instability and homelessness in the Veterans health administration. Health Serv Res. 54: 75- 85. https://doi.org/10.1111/1475-6773.13050

[4] Cohen, S.B. and Shorey, J. (2020). Artificial Intelligence and Machine Learning Derived Efficiencies for Large-Scale Survey Estimation Efforts. In Big Data Meets Survey Science https://doi.org/10.1002/9781118976357.ch19

[5] Ersoy, Mevlüt \& Şansal, Uğur. (2021). Analyze Performance of Embedded Systems with Machine Learning Algorithms. 10.1007/978-3-030-79357-9_23.

[6] Garg, Rahul \& Lamba, Shivay \& Garg, Sachin. (2021). Comparing Various Machine Learning Algorithms for
User Recommendations Systems. 10.1002/9781119752134.ch13.

[7] Guida, T. and Coqueret, G. (2018), Machine Learning in Systematic Equity Allocation: A Model Comparison. Wilmott, $2018.24-33$ https://doi.org/10.1002/wilm.10719

[8] Gilliland, M. Tashman, L. and Sglavo, U. (2021) Forecasting Methods: Modeling, Selection, and Monitoring. In Business Forecasting. https://doi.org/10.1002/9781119782605.ch3

[9] Ihya, R., Mohammed N., Guerss, F. and Haddani, H. (2020). Using Machine Learning Algorithms to Predict the E-orientation Systems Acceptancy. 10.1007/978-3030-37629-1_10.

[10] Kaun, C., Zaman, N., Goh, W. \& Sukumaran, S. (2021). Implementation of Decision Tree Algorithm to Classify Knowledge Quality in a Knowledge Intensive System. MATEC Web of Conferences. 335. 04002. 10.1051/matecconf/202133504002.

[11] Klerk, A. M. (2017). Decision Making under Uncertainty and Risk: A Retiree's Perspective on Choosing between Pension Options. Portland International Conference on Management of Engineering and Technology (PICMET), 1-12, doi: 10.23919/PICMET.2017.8125309.

[12] Li, C. Chu, D. Xu, X. and Bu, Y. (2019). A User Profile Based Pension Service Recommendation Algorithm. IEEE World Congress on Services, 374-375, doi: 10.1109/SERVICES.2019.00110.

[13] Momoh, Favour \& Rakshit, Sandip \& Vajjhala, Narasimha. (2021). Exploratory Study of Machine Learning Algorithms in Recommender Systems.

[14] Meneu Gaya R. (1996). La teoría del control óptimo en tiempo discreto. Modelos financieros aplicados a las pensiones de jubilación. [Tesis doctoral, Universidad de Valencia]

[15] Morales Cardoso S. L. (2019). Metodología para procesos de inteligencia de negocios con mejoras en la extracción y transformación de fuentes de datos, orientado a la toma de decisiones. [Tesis doctoral, Universidad de Alicante]

[16] Polatidis, N., Kapetanakis, S. \& Pimenidis, E. (2021). Recommender Systems Algorithm Selection Using Machine Learning. 10.1007/978-3-030-80568-5_39.

[17] Prasertsom, P., Kunakorntum, I. Tulyathan, A. Cheewaruangroj, N. Pornsawangdee, K. and Pasuthip, N. (2020). Benefit Recommendation System: A Use Case for Pension Fund Data Analytics. 1st International 
Conference on Big Data Analytics and Practices

(IBDAP), 1-5, doi: 10.1109/IBDAP50342.2020.9245453.

[18] Singh, S. \& Srivastva, K. (2020). Comparative Study of Machine Learning Algorithms for Recommendation System. Journal of University of Shanghai for Science and Technology. 22. 10.51201/jusst12495.

[19] Sharma, R. (2020). Machine Learning Algorithms for building Recommender Systems. 10.1109/ICCS45141.2019.9065538.

[20] Szabó, Z. (2016). Options of micro-simulation in the modelling of the pension system and the intelligent IT security system. IEEE 17 th International Symposium on Computational Intelligence and Informatics (CINTI), 295-298, doi: 10.1109/CINTI.2016.7846421.

[21] Szabó, Z. (2017). The modelling and simulation of the pension system. IEEE 30th Neumann Colloquium (NC), 25-28, doi: 10.1109/NC.2017.8263277.

[22] Wang, M. Lin, F. and Wang, M. (2019). Some Test on Optimal Portfolio of Defined Contribution Pension with HJB Equation. 18th International Symposium on Distributed Computing and Applications for Business Engineering and Science (DCABES), pp. 132-135, doi: 10.1109/DCABES48411.2019.00040.

[23] Wang, W. Cao, Y. and Xie, S. (2016). "On the system environment of pension insurance for urban workers in Heilongjiang province". Chinese Control and Decision Conference (CCDC), pp. 3153-3156, doi: 10.1109/CCDC.2016.7531525.

[24] Yuan-Yuan, Y. Ming-Lei, S. and Nuo, W. (2018). The Construction of a Cloud Computing-Based Intelligent Pension Service Platform. International Conference on Smart Grid and Electrical Automation (ICSGEA), pp. 295-298, doi: 10.1109/ICSGEA.2018.00080.

[25] Zhang, H. and Yang, Y. (2017). Optimal Control Model of Pension Funds Under Continuous Time. 13th International Conference on Computational Intelligence and Security (CIS), pp. 350-354, doi: 10.1109/CIS.2017.00082. 